Pacific Journal of Mathematics

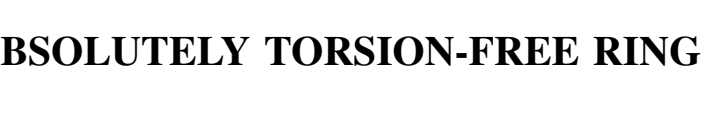




\section{ABSOLUTELY TORSION-FREE RINGS}

\section{ROBERT A. RUBIN}

Call a ring $\Lambda$ (left) absolutely torsion-free (ATF) if for every finite kernel functor $\sigma$ (i.e., a topologizing filter of nonzero left ideals), $\sigma(\Lambda)=0$. Since a commutative ring is ATF iff it is an integral domain, ATF rings may be viewed as generalizations of domains. Now an ATF ring is a prime ring, but there are even primitive rings that are not ATF. However if $A$ is either finite as a module over its center, or finite dimensional and nonsingular as a left $\Lambda$-module, then $\Lambda$ is ATF iff it is prime-in which case $\Lambda$ is right ATF as well. The class of ATF rings is closed under the formation of polynomial rings, overrings in the maximal quotient ring, and Morita equivalence, but not under subrings. If $A$ is ATF with maximal left quotient ring $Q$, then $Q$ is simple, selfinjective and von Neumann regular. Furthermore $Q$ is artinian iff $A$ is (left) finite dimensional. An interesting class of ATF rings are the hereditary noetherian prime rings (HNP). Techniques used in deriving properties of ATF rings show that every ring between an HNP ring $\Lambda$ and its maximal quotient ring is itself a ring of quotients of $A$ with respect to some idempotent kernel functor, and thus is HNP itself.

The ideas of kernel functors, filters of left ideals, torsion theories, etc."-are by now well known, and we assume the reader is familiar with them. The terminology and notation are that of Goldman [5]. In particular, for any ring $\Lambda, K(\Lambda)$ (respectively $I(\Lambda)$ ) denotes the set of kernel functors (respectively idempotent kernel functors) on the category of left $\Lambda$-modules. Furthermore, $\tau_{A}$ denotes the unique largest kernel functor with respect to which $\Lambda$ is torsion-free; $\tau_{A}$ is idempotent; a left ideal $\mathfrak{A}$ is $\tau_{\Lambda}$-open if and only if $\mathfrak{A}$ is a rational left ideal; and $Q_{\tau_{\Lambda}}(\Lambda)$ is the Utumi maximal left ring of quotients of $\Lambda$ (see [10]).

NotE. In this paper all rings have units, ring homomorphisms are unital, and the term "module" means left module over the ring being considered. Also since we are using kernel functors defined on the category of left modules, our definition is actually that of left ATF rings.

1. Absolutely torsion-free rings. The following proposition motivates our forthcoming definition.

Proposition 1.1 Let $R$ be a commutative ring. Then $R$ is an integral domain $\Leftrightarrow$ for every $\sigma \in K(R), \sigma \neq \infty \Rightarrow \sigma(R)=0$. 
Proof. $\Leftrightarrow)$ Since $\sigma(R)=\left\{x \in R \mid I x=0\right.$ for some $\left.I \in \mathscr{T}_{\sigma}\right\}$, if $\sigma \neq \infty$ (i.e., if $0 \notin \mathscr{T}_{\sigma}$ ) then $\sigma(R)=0 . \quad(\Longleftrightarrow$ ) Let $0 \neq a \in R$ and suppose $a x=0$ for some $x \in R$. Then the set of ideals $I$ of $R$ with $a \in I$ defines a kernel functor $\sigma_{a}$, and $\sigma_{a} \neq \infty$, since $a \neq 0$. But $R a$ is $\sigma_{a}$-open and $\operatorname{Rax}=0$, so $x \in \sigma_{a}(R)=0$.

Note that in the proposition above that we must check to see that $R$ has no torsion ideal for all kernel functors, not just the idempotent ones. For if we let $R=Z / p^{2} Z, p$ a prime we have: for every $\rho \in I(R), \rho \neq \infty \Rightarrow \rho(R)=0$, but $Z / p^{2} Z$ is certainly not an integral domain.

DeFinition. A ring $A$ will be called absolutely torsion-free (ATF) if for every $\sigma \in K(\Lambda), \sigma \neq \infty \Rightarrow \sigma(\Lambda)=0$.

The partial ordering on $K(\Lambda)$ provides a nice description of ATF rings.

Proposition 1.2. $A$ is absolutely torsion-free $\Leftrightarrow$ there is

$$
\mu \in I(\Lambda), \mu \neq \infty,
$$

such that for all $\infty \neq \sigma \in K(\Lambda), \sigma \leqq \mu$.

Proof. $\quad \Leftrightarrow$ Recall that $\tau_{\Lambda}$ satisfies: if $\sigma(\Lambda)=0$ then $\sigma \leqq \tau_{A}$. Since $\tau_{\Lambda} \neq \infty, \mu=\tau_{\Lambda}$ will do. $(\Leftrightarrow)$ It suffices to show that $\mu=\tau_{\Lambda}$. Now $\tau_{A} \neq \infty$ so $\tau_{A} \leqq \mu$. Hence all we must show is that $\mu \leqq \tau_{A}$; i.e., that $\mu(\Lambda)=0$. Let $I=\mu(\Lambda)$. Then $I$ is a two-sided ideal, so that the set of left ideals $\mathfrak{A}$ of $\Lambda$ such that $\mathfrak{X} \supseteqq I$ determines a kernel functor $\sigma_{I}$. If $I \neq 0$, then $\sigma_{I} \neq \infty$, and so $\sigma_{I} \leqq \mu$. Now $I$ is $\sigma_{I}$-open (i.e., $I \in \mathscr{T}_{\sigma_{I}}$ ), so $I$ is also $\mu$-open, and hence $\Lambda / I$ is $\mu$-torsion. Consider the exact sequence $0 \rightarrow I \rightarrow \Lambda \rightarrow \Lambda / I \rightarrow 0$. Since $\mu \in I(\Lambda), \Lambda$ is $\mu$-torsion, [5, Prop. 1.7]. But $\Lambda$ is $\mu$-torsion $\Rightarrow \mu=\infty$, a contradiction. Hence $\mu(\Lambda)=I=0$.

For commutative rings it is not necessary to know what a kernel functor is in order to be able to talk about integral domains. Fortunately the same is true for ATF rings, although we must introduce a new concept in order to show it.

Definition. Let $M$ be a module and $P$ a submodule of $M$. Then $P$ is said to be a weakly essential submodule of $M$, or $M$ is a weakly essential extension of $P$, if, for any finite collection $\left\{x_{1}, \cdots, x_{n}\right\}$ of elements of $M$, there is a $0 \neq r \in \Lambda$ such that $r x_{i} \in P$ for each $i=$ $1, \cdots, n$.

The following proposition describes the elementary properties of weakly essential extensions. 
Proposition 1.3. Let $P \subseteq M$. Then

(i) if $P$ is weakly essential in $M$, and if $x \in M$, then $(P: x)=$ $\{a \in \Lambda \mid a x \in P\}$ is a weakly essential left ideal of $\Lambda$;

(ii) if $P$ is an essential submodule of $M, P$ is a weakly essential submodule of $M$;

(iii) if $Q$ is an essential submodule of $P$, and if $P$ is a weakly essential submodule of $M$ then $Q$ is a weakly essential submodule of $M$.

Proof. (i ) Let $b_{1}, \cdots, b_{n}$ be elements of $\Lambda$. Then $b_{i} x \in M$ for every $i$, and since $P$ is weakly essential in $M, r b_{i} x \in P$ for every $i$, for some $0 \neq r \in \Lambda$; i.e., $r b_{i} \in(P: x)$ for every $i$. So $(P: x)$ is weakly essential.

(ii) Let $x_{1}, \cdots, x_{m} \in M$. Then for each $i=1, \cdots, m,\left(P: x_{i}\right)$ is essential, and so is $\bigcap_{i=1}^{n}\left(P: x_{i}\right)$. In particular then, $\bigcap_{i}\left(P: x_{i}\right) \neq 0$ so there is a $0 \neq r \in \Lambda$ with $r x_{i} \in P$ for each $i$.

(iii) We first show by induction that if $\left\{y_{1}, \cdots, y_{m}\right\}$ is any finite (nonempty) set of nonzero elements of $P$, there is $r \in A$ such that $r y_{i} \in Q$ for all $i$, and $r y_{i^{\prime}} \neq 0$ for some $i^{\prime}$. Since $Q$ is essential in $P$, the case $m=1$ holds. If $m>1$, there is $s \in \Lambda$ such that $0 \neq s y_{1} \in Q$. If $s y_{i} \in Q$ for all $i$ we are done. If not, the induction hypothesis applies to the nonzero elements of $\left\{s y_{2}, \cdots, s y_{m}\right\}$, and we obtain the desired element of $\Lambda$. Now if $\left\{x_{1}, \cdots, x_{n}\right\} \subseteq M$, we can find $0 \neq t \in \Lambda$ such that $t x_{j} \in P$ for each $j$. Then either each $t x_{j} \in Q$ and we are done, or we can apply the preceding to the nonzero elements of $\left\{t x_{1}, \cdots, t x_{n}\right\}$ and obtain the desired ring element.

Examples. (i) We describe weakly essential submodules for modules over rings without zero-divisors. So let $\Lambda$ be such a ring, and $P \subseteq M \Lambda$-modules. Then it is easy to check that $P$ is weakly essential in $M$ if and only if $M / P$ is torsion. For such rings we have an additional characterization of weakly essential that makes its relation to essential quite clear: $P \subseteq M$ is weakly essential if and only if whenever $N \subseteq M$ is such that $N \cap P=0$, then $N$ is torsion. Furthermore if $\Lambda$ is ATF the previous statements still hold, where "torsion" means " $\tau_{1}$-torsion".

(ii) We show that in part (iii) of Proposition 1.3 if the order of "essential" and "weakly essential" are exchanged the result is false; i.e., we give an example where $Q \subseteq P \subseteq M$ with $Q$ weakly essential in $P$ and $P$ essential in $M$, but $Q$ not weakly essential in $M$. Let $R=$ $Z / 4 Z, M=R$ as an $R$-module, $P=2 R$, and $Q=0$. Then $P$ is essential in $M$, and $Q$ is weakly essential in $P$ since $2 P \subseteq Q$. However $Q$ is not weakly essential in $M$.

The value the notion of weakly essential submodules is that it gives us a way of describing in more common language a result in- 
volving kernel functors. If $\mathfrak{A}$ is a left ideal of $\Lambda$, there is a smallest kernel functor, which we will call $\sigma_{\mathfrak{r}}$, for which $\mathfrak{A}$ is relatively open. In the following proposition we describe $\sigma_{\Re}$ via its topology.

Proposition 1.4. $\mathscr{T}_{o_{\mathfrak{U}}}$ is the set left ideals $\mathfrak{\Im}$ of $\Lambda$ such that for some finite subset $x_{1}, \cdots, x_{n}$ of $\Lambda, \mathfrak{I} \supseteqq\left(\mathfrak{X}: x_{1}\right) \cap \cdots \cap\left(\mathfrak{X}: x_{n}\right)$.

Proof. The proof that the above set of left ideals defines a kernel functor is straightforward, using only the fact that if $\mathfrak{B}$ is a left ideal, and $x$ and $y$ are elements of $\Lambda$, then $((\mathfrak{B}: x): y)=(\mathfrak{B}: y x)$. Call this kernel functor $\sigma$. Now $\mathfrak{A} \in \mathscr{T}_{\sigma}$ since $\mathfrak{U}=(\mathfrak{V}: 1)$. Furthermore if for some $\rho \in K(\Lambda), \mathfrak{N}$ is $\rho$-open, then for any finite subset $\left\{x_{1}, \cdots, x_{n}\right\}$ of $\Lambda$ each (श्V: $x_{i}$ ) is $\rho$-open and hence so is $\bigcap_{i}\left(\mathfrak{U}: x_{i}\right)$. So any $\sigma$-open left ideal is $\rho$-open; i.e., $\sigma \leqq \rho$.

Note that if $\mathfrak{A} \subseteq \mathfrak{B}$ are left ideals, then $\sigma_{\mathfrak{g}} \leqq \sigma_{\mathfrak{r}}$, and if $\rho \in K(\Lambda)$, then for any $\mathfrak{X} \in \mathscr{T}_{\rho}, \sigma_{\mathfrak{x}} \leqq \rho$. Also if $I$ is a two-sided ideal of $\Lambda$, then $\mathscr{T}_{\sigma_{I}}$ is the set of left ideals containing $I$, so $I \neq 0 \Leftrightarrow \sigma_{I} \neq \infty$.

Proposition 1.5. $\quad \sigma_{\mathfrak{r}} \neq \infty \Leftrightarrow \mathfrak{A}$ is a weakly essential left ideal.

Proof. $\quad \sigma_{\mathfrak{r}}=\infty \Leftrightarrow 0$ is $\sigma_{\mathfrak{r}}$-open $\Leftrightarrow$ for some finite subset $\left\{x_{1}, \cdots, x_{n}\right\}$ of $\Lambda, \bigcap_{i}\left(\mathfrak{X}: x_{i}\right)=0 \Leftrightarrow \mathfrak{U}$ is not weakly essential in $\Lambda$.

We can now characterize ATF rings in terms of the $\sigma_{\mathrm{g}}$ 's.

Proposition 1.6. $\Lambda$ is absolutely torsion-free $\Leftrightarrow$ the set $\mathscr{F}$ of those left ideals $\mathfrak{A}$ of $\Lambda$ such that $\sigma_{\mathfrak{x}} \neq \infty$ defines an idempotent kernel functor.

Proof. $\quad \Leftrightarrow$ Let $\mu$ be the unique largest member of $K(\Lambda)-\{\infty\}$, and note that for any left ideal $\mathfrak{A}, \sigma_{\mathfrak{r}} \neq \infty \Leftrightarrow \mathfrak{U} \in \mathscr{T}_{\mu}$. Using this fact and the remarks following Proposition 1.4, one can easily check that $\mathscr{F}=\mathscr{T}_{\mu}$.

$(\Longleftrightarrow$ If $\mathscr{F}$ defines $\rho \in I(\Lambda)$, then for any $\sigma \in K(\Lambda), \sigma \neq \infty \Rightarrow$ for any $\sigma$-open left ideal $\mathfrak{B}, \sigma_{\mathfrak{B}} \leqq \sigma$, so $\mathfrak{B}$ is $\rho$-open. Hence $\sigma \leqq \rho$, and $\Lambda$ is ATF.

If $\Lambda$ is an ATF ring, then the unique largest member of $K(\Lambda)-\{\infty\}$ as described in Proposition 1.2 is $\tau_{A}$. Recall that the elements of $\mathscr{T}_{\tau_{A}}$ are just the rational left ideals of $\Lambda$. This finally gives us a description of ATF rings that is independent of kernel functors.

THEOREM 1.7. $\Lambda$ is absolutely torsion-free $\Leftrightarrow$ every weakly essential left ideal of $\Lambda$ is a rational left ideal.

Some elementary properties of ATF rings are worth noting. 
Proposition 1.8. If $\Lambda$ is absolutely torsion-free then $\Lambda$ is a prime ring; i.e., the product of nonzero two-sided ideals of $\Lambda$ is nonzero.

Proof. Let $I, J$ be two-sided ideals of $\Lambda$. Then the set of left ideals containing $I$ defines $\sigma_{I} \in K(\Lambda)$. If $I J=0$, either $J=0$, or $\sigma_{I}(\Lambda) \neq 0$, whence $\sigma_{I}=\infty$, which can only happen if $I=0$.

Proposition 1.9. Let $\Lambda$ be absolutely torsion-free and $R$ its center. Then $R$ is an integral domain, and $\Lambda$ is a torsion-free $R$-module.

Proof. If $0 \neq a \in R$ and $y \in \Lambda$ such that $a y=0$, we have:

$$
(\Lambda a)(\Lambda y \Lambda)=\Lambda(a \Lambda)(y \Lambda)=\Lambda(\Lambda a)(y \Lambda)=\Lambda a y \Lambda=0 .
$$

Since $\Lambda$ is a prime ring, $\Lambda y \Lambda=0$, and so $y=0$.

We should have some examples of ATF rings.

(a) If $A$ has no zero divisors, $A$ is ATF. For comparing the definitions of "weakly essential" and of "rational" one sees that "weakly essential" immediately implies "rational."

(b) If $\Lambda$ is a simple ring, $\Lambda$ is ATF. This follows immediately from the fact that for any $\sigma \in K(\Lambda), \sigma(\Lambda)$ is a two-sided ideal.

(c) If $\Lambda$ satisfies the d.c.c. (on either side) then $\Lambda$ is $\operatorname{ATF} \Leftrightarrow \Lambda$ is simple. (This is the non-commutative analog of the fact that a commutative domain with d.c.c. is a field.) For if $\Lambda$ is an ATF ring with d.c.c., then $A$ is a prime ring with d.c.c., and so is simple.

Proposition 1.8 tells us that an ATF ring is a prime ring. The following example shows that the converse is false. Let $k$ be a field, and $V$ a countably infinite dimensional vector space over $k$. If $A=$ $\operatorname{Hom}_{k}(V, V)$, then $\Lambda$ is more than a prime ring; it is primitive (with $V$ as faithful simple module). Now viewing $\Lambda$ as a left $\Lambda$-module, $\Lambda$ is nonsingular (i.e., no nonzero element of $\Lambda$ has essential left annihilator) and Socle $(\Lambda) \neq 0$. Thus $\Lambda$ contains a nonsingular simple module $S$. Furthermore since $A$ is not semi-simple, $A$ has an essential maximal left ideal, and consequently a singular simple module $S^{\prime}$. If $\sigma=\tau_{S^{\prime}}$, the largest kernel functor for which $S^{\prime}$ is torsion-free, we have, since $S \approx S^{\prime}, \sigma(S)=S$ (see [5] p. 36). Thus $\sigma(\Lambda) \neq 0$, and $\Lambda$ is not ATF.

Under certain circumstances however, prime rings are ATF, when the ring is commutative for instance. We find two additional instances. The first is that the ring is "almost" commutative; i.e., it is finitely generated as a module over its center.

Proposition 1.10. Let $\Lambda$ be a ring which is finitely generated as a module over its center $R$. Then $\Lambda$ is absolutely torsion-free $\Leftrightarrow \Lambda$ is a prime ring. 
Proof. $\quad(\Leftrightarrow)$ Suppose $\sigma \in K(\Lambda)$ such that $\sigma(\Lambda) \neq 0$. Let $0 \neq x \in \sigma(\Lambda)$. Then there is a $\sigma$-open left ideal $\mathfrak{A}$ such that $\mathfrak{U} x=0$. Now $\Lambda=$ $R y_{1}+\cdots+R y_{n}$ for some $y_{1}, \cdots, y_{n} \in \Lambda$, and

$$
\mathfrak{B}=\left(\mathfrak{N}: y_{1}\right) \cap \cdots \cap\left(\mathfrak{U}: y_{n}\right) \in \mathscr{T}_{\sigma} \text {. }
$$

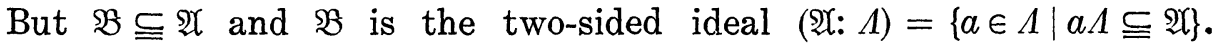
Hence $\mathfrak{B} x=0$. But then $\mathfrak{B}(\Lambda x)=(\mathfrak{V}: \Lambda) \Lambda x \subseteq \mathfrak{A} x=0$, and $x \neq 0$. So $(\mathfrak{X}: \Lambda)=0$, and hence $\sigma=\infty$.

The next instance is somewhat more interesting.

Recall that a module $M$ is called finite-dimensional, $\operatorname{dim}(M)<\infty$, if there is no infinite collection of submodules of $M$ whose sum is direct. Recall further that $z(M)=\{x \in M \mid(0: x)$ is an essential left ideal $\}$, where $(0: x)=\{r \in \Lambda \mid r x=0\}$.

TheOREM 1.11. Let $\Lambda$ be a ring such that $\operatorname{dim}\left({ }_{1} \Lambda\right)<\infty$. Then $\Lambda$ is absolutely torsion-free $\Leftrightarrow \Lambda$ is a prime ring and $z\left({ }_{\Lambda} \Lambda\right)=0$. Furthermore, if $\Lambda$ is absolutely torsion-free, it is right absolutely torsion-free as well.

Proof. $\Leftrightarrow$ Proposition 1.8 and the fact that $z \in K(\Lambda)$ yield this result. $\left(\Leftrightarrow\right.$ Let $Q=Q_{\tau_{A}}(\Lambda)$; i.e., $Q$ is the maximal left quotient ring of $A$. Then $Q$ is a simple artinian ring, and is the classical left ring of quotients of $A$ as well (Theorem 1.6 of [8]). Now since $Q$ is simple,it is ATF, and since $Q$ is semi-simple as well, the only weakly essential left ideal of $Q$ is $Q$ itself. Let $\mathfrak{A}$ be a weakly essential left ideal of $\Lambda$, and let $q_{1}, \cdots, q_{n} \in Q$. Then there is a regular $d \in \Lambda$ such that $d q_{i} \in \Lambda$ for each $i=1, \cdots, n$. Furthermore there is $0 \neq r \in \Lambda$ such that $r d q_{i} \in \mathfrak{X}$ for each $i$. Since $d$ is regular, $r d \neq 0$, and so $Q \mathcal{A}$ is a weakly essential $\Lambda$-submodule, and hence $Q$-submodule, of $Q$. Thus $Q \mathfrak{A}=Q$. Then by Lemma 1.5 of [8], $\mathfrak{O}$ is an essential left ideal of $\Lambda$. But essential left ideals of rings satisfying $z(\Lambda)=0$ are rational. Thus $\Lambda$ is ATF.

Now let $\delta$ be a kernel functor defined on the category of right $\Lambda$-modules. Suppose that $\infty \neq \delta$, and that $I=\delta(\Lambda) \neq 0$. Then $I$ is a two-sided ideal, and since $\Lambda$ is a prime ring, $I$ is essential as a left ideal. Thus $Q I=Q$ by Theorem 1.6 of [8] again, and so there are $q_{i} \in Q, x_{i} \in I, i=1, \cdots, m$ such that $1=\sum_{i} q_{i} x_{i}$. Since each $x_{i} \in I$, there is a nonzero right ideal $\Im$ of $\Lambda$ such that $x_{i} \mathfrak{\Im}=0$ for each $i$. But if $a \in \mathfrak{\Im}, a=1 \cdot a=\sum q_{i} x_{i} a=0$. Thus $\mathfrak{\Im}=0$, a contradiction. Hence $I=0$, and $A$ is right ATF.

Note. While the definition of ATF rings is one-sided, the two preceding results give rather common situations under which the 
condition is actually two-sided. This naturally raises the question as to whether there are any one-sided ATF rings.

Given a commutative domain $R$, it is easy to make other domains: subrings of $R$, rings between $R$ and its field of quotients, polynomial rings over $R$ are all domains. We now turn to question of obtaining new "domains" from an old one in the more general setting. The following theorem, which is of considerable interest in itself, immediately yields an important fact about ATF rings.

THEOREM 1.12 Let $\Lambda$ and $\Gamma$ be rings, and suppose that the category of (left) $\Lambda$-modules, $\mathscr{C}(\Lambda)$, is equivalent to the category of (left) $\Gamma$-modules, $\mathscr{C}(\Gamma)$; i.e., there are functors $S: \mathscr{C}(\Lambda) \rightarrow \mathscr{C}(\Gamma)$ and $T: \mathscr{L C}(\Gamma) \rightarrow \mathscr{C}(\Lambda)$ such that $S T$ (resp. TS) is naturally equivalent to the identity functor on $\mathscr{C}(\Gamma)($ resp. $\mathscr{C}(\Lambda))$. Then $K(\Gamma)$ is isomorphic to $K(\Lambda)$ as partially ordered sets.

Proof. Define $S: K(\Lambda) \rightarrow K(\Gamma)$ and $T: K(\Gamma) \rightarrow K(\Lambda)$ by: if $\sigma \in K(\Lambda)$ and if $X$ is a $\Gamma$-module, then $X$ is $S(\sigma)$-torsion $\Leftrightarrow X \approx S(M)$ for some $\sigma$-torsion $\Lambda$-module $M$; similarly if $\rho \in K(\Gamma)$ and $M$ is a $\Lambda$-module, call $M T(\rho)$-torsion $\Leftrightarrow M \approx T(X)$ for some $\rho$-torsion $\Gamma$-module $X$. It is routine that $S$ and $T$ are order preserving and inverses of each other.

CoRollary 1.13. If $\Lambda$ is absolutely torsion-free, and $\Gamma=M_{n}(\Lambda)$, the $n \times n$ matrix ring with entries in $\Lambda$, then $\Gamma$ is absolutely torsion-free.

Proof. As is well known, the category of left $\Lambda$-modules, $\mathscr{C}(\Lambda)$, is equivalent to the category of left $M_{n}(\Lambda)$-modules, $\mathscr{C}_{n}(\Lambda)$. So $K(\Lambda)$ and $K\left(M_{n}(\Lambda)\right)$ are the same as partially ordered sets. But by Proposition 1.2, the condition for being ATF is stated purely in terms of the partial ordering on $K(\Lambda)$. So $M_{n}(\Lambda)$ is ATF as well.

The next theorem shows that the formation of polynomial rings preserves our condition.

THEOREM 1.14. Let $\Lambda$ be absolutely torsion-free and $\Gamma=\Lambda[x]$, i.e., we adjoint to $A$ a central nonzero-divisor, nonunit $x$. Then $\Gamma$ is absolutely torsion-free.

Proof. If $\infty \neq \sigma \in K(\Gamma)$, and $g \in \sigma(\Gamma)$, then $g$ is annihilated by a weakly essential left ideal. Thus it suffices to show that no weakly essential left ideal of $\Gamma$ annihilates any nonzero element of $\Gamma$. Let $\mathfrak{X}$ be any left ideal of $\Gamma$, and let $\mathfrak{X}=\left\{r \in \Lambda \mid a_{0}+a_{1} x+\cdots+\right.$ $a_{n-1} x^{n-1}+r x^{n} \in \mathfrak{X}$ for some $\left.a_{0}, \cdots, a_{n-1} \in \Lambda\right\}$ i.e., $\mathfrak{X}$ is the set of "highest coefficients of $\mathfrak{X} . "$ Then $\mathfrak{A}$ is a left ideal of $\Lambda$. Further- 
more, if $\mathfrak{X}$ is a weakly essential left ideal of $\Gamma$, then $\mathfrak{A}$ is weakly essential in $\Lambda$, for if $z_{1}, \cdots, z_{p}$ is any finite subset of $\Lambda$, then $z_{1}, \cdots, z_{p}$ is also a finite subset of $\Gamma$, and so there is a $0 \neq f \in \Gamma, f=a_{0}+$ $a_{1} x+\cdots+a_{n} x^{n}, a_{n} \neq 0$, such that $f z_{i} \in \mathfrak{X}$ for each $i$. This means that $a_{0} z_{i}+a_{i} z_{i} x+\cdots+a_{n} z_{i} x^{n} \in \mathfrak{X}$ for each $i$, and so $a_{n} z_{i} \in \mathfrak{X}$ for each $i$, and $a_{n} \neq 0$. Suppose now that $\mathfrak{X}$ is weakly essential in $\Gamma$, and that $\mathfrak{X} g=0$, for some $g=b_{0}+b_{1} x+\cdots+b_{m} x^{m} \in \Gamma$. Now if $\mathfrak{A}$ is the set of highest coefficients of $\mathfrak{X}$ as above, let $a \in \mathfrak{X}$. Then $h=c_{0}+c_{1} x+\cdots+a x^{p} \in \mathfrak{X}$ and $h g=0$. Now the highest coefficient of $h g$ is $a b_{m}$, the coefficient of $x^{m+p}$. So $\mathfrak{U} b_{m}=0$ and since $\mathfrak{X}$ is weakly essential, and hence rational in $\Lambda, \mathfrak{N} b_{m}=0 \Rightarrow b_{m}=0$. Now $b_{m}$ is the highest coefficient of $g$, so $g=0$.

Next we show that all rings between an ATF ring and its maximal quotient ring are still ATF.

THEOREM 1.15. Let $\Lambda$ be absolutely torsion-free and let $Q=Q_{\tau_{\Lambda}}(\Lambda)$. Then if $\Gamma$ is a ring such that $\Lambda \leqq \Gamma \leqq Q$, then $\Gamma$ is absolutely torsion-free.

Proof. Let $\mathfrak{X}$ be a weakly essential left ideal of $\Gamma$, and let $\mathfrak{A}=\Lambda \cap \mathfrak{X}$. Then $\mathfrak{A}$ is a left ideal of $\Lambda$, and if $x_{1}, \cdots, x_{n}$ is any finite subset of $A$, it is also a finite subset of $\Gamma$, so there exists $0 \neq \gamma \in \Gamma$ with $\gamma x_{i} \in \mathfrak{X}$ for each $i$. However $0 \neq \gamma \in Q$, so, since $Q / \Lambda$ is $\tau_{A}$-torsion while $Q$ is $\tau_{A}$-torsion-free, $0 \neq r \gamma \in \Lambda$ for some $r \in \Lambda$. Then $(r \gamma) x_{i} \in \mathfrak{X} \cap \Lambda$ for each $i$, i.e., $\mathfrak{X}$ is weakly essential, and so is rational in $\Lambda$. By noting $\mathfrak{A} \subset \Lambda$, that $\Lambda$ is rational in $Q$, and that $\mathfrak{A} \subseteq \mathfrak{X} \subseteq \Gamma \leqq Q$, and finally that rationality is transitive, [10, 1.5, pg. 2] we see that $\mathfrak{X}$ is rational in $\Gamma$. Hence by Theorem $1.7 \Gamma$ is ATF.

We have seen a number of ways in which new ATF's naturally arise from a given one. There is one manner in which they fail to arise, despite what occurs in the commutative case; namely from taking subrings.

Let $\Lambda$ be the ring of $2 \times 2$ lower triangular matrices over a field $k$. Then the maximal ring of quotients of $\Lambda$ is $M_{2}(k)$, the $2 \times 2$ full matrix ring. $M_{2}(k)$ is ATF while $\Lambda$, which has a nilpotent ideal, is not. So it can happen that very reasonable subrings of ATF rings need not be themselves ATF. An interesting problem would be to determine which subrings of ATF rings are ATF.

2. The maximal ring of quotients of an ATF ring. For any ring $\Lambda$, Utumi's maximal left ring of quotients is $Q_{\tau_{\Lambda}}(\Lambda)$ in the language of [5] (see [10]). In this section we describe the maximal (left) ring of quotients of an ATF ring. 
THEOREM 2.1. Let $\Lambda$ be an absolutely torsion-free ring, and $Q\left(=Q_{2}(\Lambda)\right)$ its maximal left ring of quotients. Then $Q$ is simple, von Neumann regular, and self-injective (as left Q-module). Furthermore $Q$ satisfies the d.c.c. $\Leftrightarrow \Lambda$ is (Goldie) finite dimensional as a left A-module.

Proof. Since an ATF ring is nonsingular, all but the simplicity of $Q$ is well known: that $Q$ is von Neumann regular goes all the way back to Johnson [6]; [Prop. 3, p. 95 and the Corollary to Prop. 2, pg. 106 of 7] applied to $Q$ itself yields self injectivity; and, once we have simplicity, the furthermore is taken care of by Theorem 1.6 of [8]. We give a proof due to Goldman that $Q$ is simple.

For any module $M$ over any ring, there is a smallest kernel functor, which we will call $\nu_{M}$, with respect to which $M$ is torsion. Furthermore $\mathscr{T}_{\nu_{M}}$ is the set of left ideals containing some finite intersection of annihilators of elements of $M$. Let $I$ be a two-sided ideal of $Q$, which by Theorem 1.15 is ATF. If $I \neq 0$, we have, since $\nu_{I}(Q) \supseteqq \nu_{I}(I)=I \neq 0, \nu_{I}=\infty$. Hence there are elements $x_{1}, \cdots, x_{n} \in I$ such that ann $\left(x_{1}\right) \cap \cdots \cap$ ann $\left(x_{n}\right)=0$, where ann $\left(x_{j}\right)=\left\{q \in Q \mid q x_{j}=0\right\}$. Let $I^{n}=I \oplus \cdots \oplus I$ ( $n$ times) and let $f: Q \rightarrow I^{n}$ be defined by $f(1)=$ $\left(x_{1}, x_{2}, \cdots, x_{n}\right)$. Then $f$ is a $Q$-monomorphism from $Q$ to $I^{n}$, and since $Q$ is injective, $f$ splits; i.e., there is a $Q$-homomorphism $g: I^{n} \rightarrow Q$ such that $g f(1)=1$. Now $g$ is a map from a sum, so there are $Q$-homomorphisms $g_{j}: I \rightarrow Q, j=1, \cdots, n$, such that for any $\left(a_{1}, \cdots, a_{n}\right) \in I^{n}$ $g\left(\left(a_{1}, \cdots, a_{n}\right)\right)=\sum_{j} g_{j}\left(a_{j}\right)$. But again $Q$ is injective, and $I$ is a left ideal of $Q$. Hence for each $j=1, \cdots, n$ there is $q_{j} \in Q$ such that $g_{j}(a)=a q_{j}$ for any $a \in I$. Thus $1=g(f(1))=g\left(\left(x_{1}, \cdots, x_{n}\right)\right)=\sum_{j} g_{j}\left(x_{j}\right)=$ $\sum_{j} x_{j} q_{j}$. But each $x_{j} \in I$, which is a two-sided ideal of $Q$. Thus either $I=0$, or $1 \in I$. Hence $Q$ is simple.

3. Hereditary noetherian prime rings. Recall that a ring is left hereditary if every left ideal is projective. Recently a great deal of interest has been shown in hereditary noetherian prime rings, these being very reasonable noncommutative analogs of dedekind domains. Here hereditary and noetherian are assumed to hold on both sides. See [2] and [3] for more about these rings. In this section, we shall show that every overring of an hereditary noetherian prime ring $\Lambda$ in its maximal quotient ring is of the form $Q_{\sigma}(\Lambda)$ for some $\sigma \in I(\Lambda)$, and is itself hereditary noetherian and prime. In doing so we shall have need of the following restatement of Theorem 13.10 of [9].

THEOREM 3.1. Let $\Lambda \stackrel{f}{\rightarrow} \Gamma$ be an epimorphism of rings. If $f$ induces on $\Gamma$ the structure of a flat right 1-module, then there is a $\mu \in I(\Lambda)$ such that $\Gamma \approx Q_{\mu}(\Lambda)$. 
THEOREM 3.2. Let $\Lambda$ be an hereditary noetherian prime ring and $Q$ its maximal ring of quotients. Let $\Gamma$ be a ring such that $\Lambda \subseteq \Gamma \leqq Q$. Then there is a $\mu \in I(\Lambda)$ such that $\Gamma \approx Q_{\mu}(\Lambda)$.

Proof. By Theorem 3.1 it suffices to show that the inclusion of $A$ in $\Gamma$ is an epimorphism of rings and induces on $\Gamma$ the structure of a fiat right $\Lambda$-module. Now by Theorem 2.1 of [2], every finitely generated right $\Lambda$-submodule of $Q$ is projective (since the $t$ of that theorem is $\tau_{A}$ in our language, and $\tau_{A}(Q)=0$ ). But projective right $\Lambda$-modules are right flat, $\Gamma$ is the direct limit of its finitely generated right $\Lambda$-submodules, and flatness is preserved by direct limits. Hence $\Gamma$ is flat as a right $\Lambda$-module. Now since $\Gamma \subseteq Q$ and $\Gamma$ is right $\Lambda$-flat, $\Gamma \otimes_{A} \Gamma \subseteq \Gamma \otimes_{1} Q$. But $Q$ is itself right $\Lambda$-flat (Theorem 2.7 of [8]), so tensoring $\Gamma \leqq Q$ with $Q$ gives $Q \otimes_{A} \Gamma \leqq Q \otimes_{4} Q$. But it is wellknown that $Q \otimes_{A} Q \approx Q$. For instance Theorem 4.3 of [5], and the fact that $Q$ can be viewed either as $Q_{\tau_{A}}(\Lambda)$ or as the classical ring of quotients of $\Lambda$, yield this quickly. So $\Gamma \otimes_{1} \Gamma \subseteq Q \otimes_{1} Q \approx Q$. But the image of $\Gamma \otimes_{1} \Gamma$ in $Q$ is just $\Gamma$; i.e., $\Gamma \otimes_{A} \Gamma \approx \Gamma$, so $A \subseteq \Gamma$ is an epimorphism of rings.

The above theorem allows us to prove the noncommutative analog of the theorem that says every ring between a Dedekind domain and its quotient field is also Dedekind. Some preliminaries are needed however, including the following well-known lemma.

Lemma 3.3. Let $\sigma \in I(\Lambda), S$ and $T Q_{o}(\Lambda)$-modules, and $f$ a $\Lambda$-homomorphism from $S$ to $T$. Then if $\sigma(T)=0$ when $T$ is considered as a A-module, $f$ is a $Q_{\sigma}(\Lambda)$-homomorphism.

Proof. Let $q \in Q_{\sigma}(\Lambda)$ and $x \in S$. Then for some $\mathfrak{X} \in \mathscr{T}_{\sigma}, \mathfrak{A} q \subset i(\Lambda)$. Then for any $a \in \mathfrak{N}, a f(q x)=f(a q x)=(a q) f(x)$ so $a(f(q x)-q f(x))=0$. Hence $\mathfrak{A}(f(q x)-q f(x))=0 \Rightarrow f(q x)=q f(x) \in \sigma(T)=0$. Hence $f(q x)=$ $q f(x)$ for all $x \in S$ and $q \in Q_{\sigma}(\Lambda)$; i.e., $f$ is a $Q_{\sigma}(\Lambda)$-homomorphism.

Proposition 3.4. Let $\sigma \in I(\Lambda)$, and suppose $\Lambda \subseteq Q_{o}(\Lambda)$; i.e., $\sigma(\Lambda)=0$. Let $\mathfrak{X}$ be a left ideal of $Q_{o}(\Lambda)$, and $\mathfrak{A}=\Lambda \cap \mathfrak{X}$. Then if $\mathfrak{X}$ is a finitely generated projective 1 -module, $\mathfrak{X}$ is a finitely generated projective $Q_{o}(\Lambda)$-module.

Proof. Recall that a module $M$ is projective if and only if there exist families $\left\{x_{\alpha}\right\}$ of elements of $M$ and $\Lambda$-homomorphism $\left\{f_{\alpha}: M \rightarrow \Lambda\right\}$ such that for each $x \in M$, almost all $f_{\alpha}(x)=0$ and $x=\sum_{\alpha} f_{\alpha}(x) x_{\alpha}$ [1, Prop. 3.1, p. 132]. Furthermore $M$ is finitely generated projective if and only if the families $\left\{x_{\alpha}\right\}$ and $\left\{f_{\alpha}\right\}$ are finite. So now let $\mathfrak{X}$ be a left ideal of $Q_{\sigma}(\Lambda)$ such that $\mathfrak{X}=\mathfrak{X} \cap \Lambda$ is finitely generated pro- 
jective. Then we have finite families $\left\{x_{\alpha}\right\} \subseteq \mathfrak{X}$ and $\left\{f_{\alpha}: \mathfrak{U} \rightarrow \Lambda\right\}$ such that for any $a \in \mathfrak{A}, a=\sum_{\alpha} f_{\alpha}(\alpha) x_{\alpha}$. Now let $x \in \mathfrak{X}$; then $x \in Q_{o}(\Lambda)$, so $\Im x \leqq \Lambda$ for some $\Im \in \mathscr{T}_{\sigma^{*}}$ But $\mathfrak{T} \subseteq \cong \mathfrak{X}$ as well, so $\mathfrak{F} \subset \Lambda \cap \mathfrak{X}=\mathfrak{A}$. Hence $\mathfrak{X} / \mathfrak{X}$ is $\sigma$-torsion. For any $\alpha$, we obtain the following diagram of $\Lambda$-modules and $\Lambda$-maps:

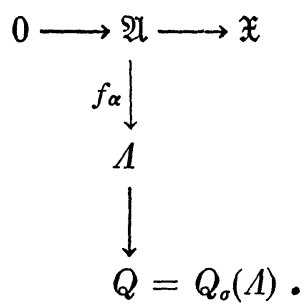

Since $Q$ is $\sigma$-torsion-free and $\sigma$-injective there is a unique $\Lambda$-homomorphism $g_{\alpha}: \mathfrak{X} \rightarrow Q$ making the diagram commute. The preceding lemma shows that $g_{\alpha}$ is a $Q$-homomorphism. Now define, for any $x \in \mathfrak{X}, g(x)=\sum_{\alpha} g_{\alpha}(x) x_{\alpha}$. Note that $g$ is a $Q$-homomorphism, and hence a $\Lambda$-homomorphism, from $\mathfrak{X}$ to $Q$ and that $g$ restricts to the identity on $\mathfrak{A}$. Consider this diagram of $\Lambda$-modules:

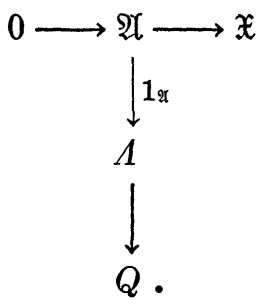

Now there is a unique $\Lambda$-homomorphism $h$, which again is a $Q$-homomorphism, making the diagram commute. We could certainly have $h=1_{x}$, and we could also have $h=g$. So $g=1_{x}$; i.e., for any $x \in \mathfrak{X} x=$ $g(x)=\sum_{\alpha} g_{\alpha}(x) x_{\alpha}$ so $\mathfrak{X}$ is finitely generated projective as a $Q_{o}(\Lambda)$-module.

THEOREM 3.5. Let $\Lambda$ be an hereditary noetherian prime ring with maximal ring of quotients $Q\left(\approx Q_{\tau_{\Lambda}}(\Lambda)\right)$. If $\Gamma$ is a ring with $\Lambda \subseteq \Gamma \leqq Q, \Gamma$ is an hereditary noetherian prime ring.

Proof. By Theorem 1.15 $\Gamma$ is ATF, hence prime. Theorem 3.2 says that $\Gamma \approx Q_{\sigma}(\Lambda)$ some $\sigma \in I(\Lambda)$, and so by Proposition 3.4. $\Gamma$ is left noetherian and left hereditary. But since $Q$ is the two sided classical ring of quotients of $\Lambda, \Gamma$ is also left $\Lambda$-flat, and Theorem 3.2 holds for $\Gamma$ as a right ring of quotients of $\Lambda$. So Proposition 3.4 applies on the right, and $\Gamma$ is right hereditary and right noetherian as well; i.e., $\Gamma$ is an hereditary noetherian prime ring. 
REMARK. Using the language of Theorem 4.3 of of [5] we can readily show that if $\sigma, \rho$ are kernel functors having Property (T), then $Q_{\sigma}(\Lambda) \approx Q_{\rho}(\Lambda) \Rightarrow \sigma=\rho$. From this a generalization of a fact about Dedekind domains can be made: if $\Lambda$ is an hereditary noetherian prime ring, and $\sigma, \rho \in I(\Lambda)$, then $\sigma \neq \rho \Rightarrow Q_{\sigma}(\Lambda) \approx Q_{\rho}(\Lambda)$.

\section{REFERENCES}

1. H. Cartan and S. Eilenberg, Homological Algebra, Princeton Univ. Press, 1956.

2. D. Eisenbud and J. C. Robson, Modules over Dedekind prime rings, J. Algebra, 16 (1970), 67-85.

3. - Hereditary Noetherian prime rings, J. Algebra, 16 (1970), 86-104.

4. A. W. Goldie, The structure of prime rings under ascending chain conditions, Proc. London Math. Soc., 8 (1958), 589-608.

5. O. Goldman, Rings and modules of quotients, J. Algebra, 13 (1969), 10-47.

6. R. E. Johnson, The extended centralizer of a ring over a module, Proc. Amer. Math. Soc., $\mathbf{z}$ (1951), 891-895.

7. J. Lambek, Lectures on Rings and Modules, Blaisdell, Waltham, Mass. (1966).

8. F. L. Sandomierski, Semi-simple maximal quotient rings, Trans. Amer. Math. Soc., 128 (1967), 112-120.

9. B. Stenstrom, Rings and Modules of Quotients, Springer Lecture Notes 237, 1971.

10. Y. Utumi, On quotient rings, Osaka Math. J., 8 (1956), 1-18.

Received February 3, 1972 and in revised form July 28, 1972. This paper constitutes a portion of the author's doctoral dissertation at the University of Pennsylvania. The author wishes to express his deep gratitude to Professor Oscar Goldman, his advisor, for the guidance and inspiration he provided. To Professor Vasily C. Cateforis thanks are due for many fruitful conversations.

UNIVERSITY OF KENTUCKY 


\section{PACIFIC JOURNAL OF MATHEMATICS}

\section{EDITORS}

D. Gilbarg and J. Milgram

Stanford University

Stanford, California 94305

\author{
R. A. Beaumont \\ University of Washington \\ Seattle, Washington 98105
}

J. DuGUNDJI

Department of Mathematics

University of Southern California

Los Angeles, California 90007

Richard ARENS

University of California

Los Angeles, California 90024

\section{ASSOCIATE EDITORS}
E. F. BECKENBACH
B. H. NeumanN
F. WOLF
K. YosHIDA

\section{SUPPORTING INSTITUTIONS}

UNIVERSITY OF BRITISH COLUMBIA

CALIFORNIA INSTITUTE OF TECHNOLOGY

UNIVERSITY OF CALIFORNIA

MONTANA STATE UNIVERSITY

UNIVERSITY OF NEVADA

NEW MEXICO STATE UNIVERSITY

OREGON STATE UNIVERSITY

UNIVERSITY OF OREGON

OSAKA UNIVERSITY

\author{
UNIVERSITY OF SOUTHERN CALIFORNIA \\ STANFORD UNIVERSITY \\ UNIVERSITY OF TOKYO \\ UNIVERSITY OF UTAH \\ WASHINGTON STATE UNIVERSITY \\ UNIVERSITY OF WASHINGTON
* * * *
AMERICAN MATHEMATICAL SOCIETY \\ NAVAL WEAPONS CENTER
}

The Supporting Institutions listed above contribute to the cost of publication of this Journal, but they are not owners or publishers and have no responsibility for its content or policies.

Mathematical papers intended for publication in the Pacific Journal of Mathematics should be in typed form or offset-reproduced, (not dittoed), double spaced with large margins. Underline Greek letters in red, German in green, and script in blue. The first paragraph or two must be capable of being used separately as a synopsis of the entire paper. The editorial "we" must not be used in the synopsis, and items of the bibliography should not be cited there unless absolutely necessary, in which case they must be identified by author and Journal, rather than by item number. Manuscripts, in duplicate if possible, may be sent to any one of the four editors. Please classify according to the scheme of Math. Rev. Index to Vol. 39. All other communications to the editors should be addressed to the managing editor, Richard Arens, University of California, Los Angeles, California, 90024.

50 reprints are provided free for each article; additional copies may be obtained at cost in multiples of 50 .

The Pacific Journal of Mathematics is issued monthly as of January 1966. Regular subscription rate: $\$ 48.00$ a year (6 Vols., 12 issues). Special rate: $\$ 24.00$ a year to individual members of supporting institutions.

Subscriptions, orders for back numbers, and changes of address should be sent to Pacific Journal of Mathematics, 103 Highland Boulevard, Berkeley, California, 94708.

PUBLISHED BY PACIFIC JOURNAL OF MATHEMATICS, A NON-PROFIT CORPORATION

Printed at Kokusai Bunken Insatsusha (International Academic Printing Co., Ltd.), 270, 3-chome Totsuka-cho, Shinjuku-ku, Tokyo 160, Japan. 


\section{Pacific Journal of Mathematics}

\section{Vol. 46, No. 2 December, 1973}

Christopher Allday, Rational Whitehead products and a spectral sequence of

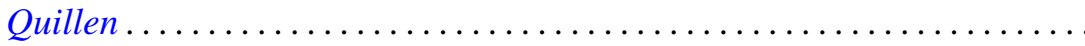

James Edward Arnold, Jr., Attaching Hurewicz fibrations with fiber

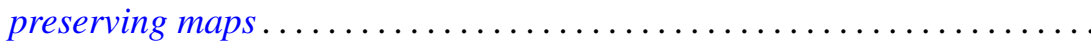

Catherine Bandle and Moshe Marcus, Radial averaging transformations with various metrics.................................

David Wilmot Barnette, A proof of the lower bound conjecture for convex

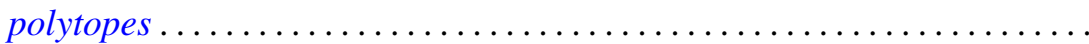

Louis Harvey Blake, Simple extensions of measures and the preservation of

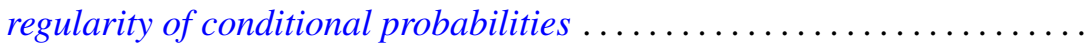

James W. Cannon, New proofs of Bing's approximation theorems for

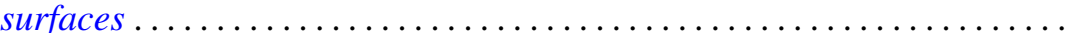

C. D. Feustel and Robert John Gregorac, On realizing HNN groups in

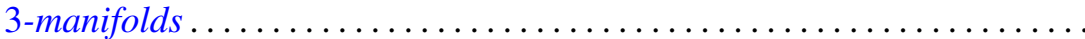

Theodore William Gamelin, Iversen's theorem and fiber algebras . . . . . . . . 389

Daniel H. Gottlieb, The total space of universal fibrations . . . . . . . . . . . .

Yoshimitsu Hasegawa, Integrability theorems for power series expansions of

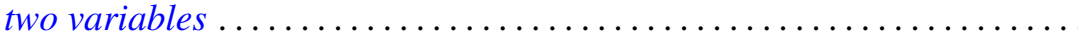

Dean Robert Hickerson, Length of period simple continued fraction expansion of $\sqrt{ } d$

Herbert Meyer Kamowitz, The spectra of endomorphisms of the disc algebra.

Dong S. Kim, Boundedly holomorphic convex domains

Daniel Ralph Lewis, Integral operators on $\mathscr{L}_{p}$-spaces ...

John Eldon Mack, Fields of topological spaces . . . . . . . . .

V. B. Moscatelli, On a problem of completion in bornology

Ellen Elizabeth Reed, Proximity convergence structures. .

Ronald C. Rosier, Dual spaces of certain vector sequence spaces .

Robert A. Rubin, Absolutely torsion-free rings

Leo Sario and Cecilia Wang, Radial quasiharmonic functions . .

James Henry Schmerl, Peano models with many generic classes .

H. J. Schmidt, The $\mathscr{F}$-depth of an $\mathscr{F}$-projector ............

Edward Silverman, Strong quasi-convexity. . . . . . . . . . . . . . . . . 549

Barry Simon, Uniform crossnorms ....................... 555

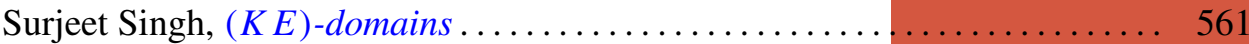

Ted Joe Suffridge, Starlike and convex maps in Banach spaces . . . . . . . . 575

Milton Don Ulmer, $C$-embedded $\Sigma$-spaces . . . . . . . . . . . . . . . . 591

Wolmer Vasconcelos, Conductor, projectivity and injectivity . . . . . . . . . 603 